\title{
CoBiD-net: a tailored deep learning ensemble model for time series forecasting of covid-19
}

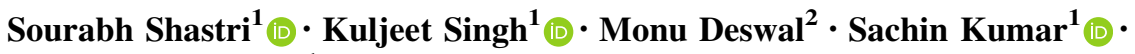 \\ Vibhakar Mansotra ${ }^{1}$
}

Received: 5 February 2021/Revised: 19 May 2021 / Accepted: 30 May 2021 / Published online: 12 June 2021

(C) Korean Spatial Information Society 2021

\begin{abstract}
The pandemic of novel coronavirus disease 2019 (Covid-19) has left the world to a standstill by creating a calamitous situation. To mitigate this devastating effect the inception of artificial intelligence into medical health care is mandatory. This study aims to present the educational perspective of Covid-19 and forecast the number of confirmed and death cases in the USA, India, and Brazil along with the discussion of endothelial dysfunction in epithelial cells and Angiotensin-Converting Enzyme 2 receptor (ACE2) with the Covid-19. Three different deep learning based experimental setups have been framed to forecast Covid-19. Models are (i) Bi-directional Long Short Term Memory (LSTM) (ii) Convolutional LSTM (iii) Proposed ensemble of Convolutional and Bi-directional LSTM network are known as CoBiD-Net ensemble. The educational perspective of Covid-19 has been given along with an
\end{abstract}

Supplementary Information The online version contains supplementary material available at https://doi.org/10.1007/s41324021-00408-3.

Kuljeet Singh

kuljeetshan94@gmail.com

Sourabh Shastri

sourabhshastri@gmail.com

Monu Deswal

monudeswal19@gmail.com

Sachin Kumar

skh.sachinkumar@gmail.com

Vibhakar Mansotra

vibhakar20@yahoo.co.in

1 Department of Computer Science and IT, University of Jammu, Jammu, Jammu \& Kashmir 180006, India

2 All India Institute of Medical Sciences (AIIMS), New Delhi, India architectural discussion of multi-organ failure due to intrusion of Covid-19 with the cell receptors of the human body. Different classification metrics have been calculated using all three models. Proposed CoBiD-Net ensemble model outperforms the other two models with respect to accuracy and mean absolute percentage error (MAPE). Using CoBiD-Net ensemble, accuracy for Covid-19 cases ranges from 98.10 to $99.13 \%$ with MAPE ranges from 0.87 to 1.90 . This study will help the countries to know the severity of Covid-19 concerning education in the future along with forecasting of Covid-19 cases and human body interaction with the Covid-19 to make it the self-replicating phenomena.

Keywords Education · LSTM · Deep learning · Forecasting · Endothelial dysfunction · Covid-19

\section{Introduction}

In December 2019 the catastrophic phase of the twentyfirst century has begun in the form of Novel Coronavirus (Covid-19) which ravages the year 2020 in all terms. Initially, it has been started in Wuhan, the city of China and within a couple of months, it grabs the world under its disastrous network. In March 2020 World Health Organization (WHO) has declared novel coronavirus disease 2019 (Covid-19) as a global pandemic. As of 30th April 2021 WHO recorded that this highly contagious virus has claimed over 3,169,494 lives with 150,699,791 confirmed cases in 220 countries and territories in the world. Transmission classification along with the number of confirmed and death cases in the USA, India, and Brazil are maximum than other nations shown in Table 1 [1]. The Covid-19 has almost impacted everything the nations stand on such as 
Table 1 Covid-19 spread analysis of world and top three affected countries (accessed on April 20, 2021)

\begin{tabular}{llllll}
\hline Name & $\begin{array}{l}\text { Cumulative } \\
\text { cases }\end{array}$ & $\begin{array}{l}\text { Cases- newly reported in last } \\
24 \mathrm{~h}\end{array}$ & $\begin{array}{l}\text { Cumulative } \\
\text { deaths }\end{array}$ & $\begin{array}{l}\text { Deaths- newly reported in last } \\
24 \mathrm{~h}\end{array}$ & $\begin{array}{l}\text { Transmission } \\
\text { classification }\end{array}$ \\
\hline Global & $141,754,944$ & 656,406 & $3,025,835$ & 10,503 & - \\
USA & $31,350,025$ & 38,084 & 561,921 & 305 & Community transmission \\
India & $15,321,089$ & 259,170 & 180,530 & 1,761 & Cluster of Cases \\
Brazil & $13,943,071$ & 42,980 & 373,335 & 1,657 & Community transmission \\
\hline
\end{tabular}

the production, economy, and education. Covid-19 has also shaken the base of the sustainable development goal agenda of 2030 targeted by the United Nations (UN). Due to the pandemic situation goals like no poverty, zero hunger, good health and well being, and quality education are at greater threat. Quality education makes nations strong than stronger, but due to the current situation of the pandemic, this looks too hard to make such a standard of quality education [2]. For 10 months almost all educational institutions are closed to limit the spread of Covid-19 from community transmission, although the USA and Brazil are already struggling from the phase of community transmission and India is at greater risk.

Due to the partial closure of educational institutions for such a long period, the educational sector has been shifted its working from offline to online mode overnight. Therefore to sustain the academic progress of the student's E-Learning became the mandatory part of the curriculum. Although there are certain regions/states in each country where internet connectivity is very poor and school buildings are turned into quarantine centers. Union Territory of Jammu and Kashmir, India is one such example where high-speed internet connectivity is banned since mid-2019. To carry out online classes and research in such a scenario is quite a difficult task for all academicians. This unbearable loss that has been faced by the students/scholars due to improper availability of resources can't be filled. Almost all the countries have been suffered from continuous lockdown and restrictions of Covid-19. The hillbilly region of southern states in the USA, Rochina in Rio Brazil, and Khayelitsha region of South Africa are some of those regions that are suffered most due to Covid-19 in respect of lack in education due to poor internet connectivity and unavailability of daily necessities of life [3]. People living in poverty have faced unconditional problems at most. Persons who are daily wagers to support their families have been crushed due to this pandemic because they have no access to information and communication technology to pursue their online learning and don't have any stores of daily feed requirements to live with. As the catastrophic phase of Covid-19 has turned the tables over, the more responsibility that policymakers and researchers have to find a way out of this pandemic and suggest some sustainable strategies to open learning accessibility as before. To mitigate the Covid-19 effect, policymakers are applying the following two strategies that are not sufficient for densely populated nations like India. Move testing facilities to the doorstep of individuals and to build different Covid-19 care centers where individuals have to go for testing.

The first step is hardly feasible and costly to implement in real-time. The second step is feasible but a bit risky and hectic for those who are not infected by Covid-19 [4]. There must exist some experimental study to predict the future effect of Covid-19 for most affected nations so that policymakers can accordingly subjugate the Covid-19 effect down to the end. One such study is proposed in this research article. In this paper, deep learning algorithms are used to design the proposed model, a recurrent neural network (RNN) based Long Short Term Memory (LSTM) method is used to actually design the proposed ensemble model.

The rest of the paper is organized as follows: Sect. 2 represents the review of recent scholarly works that are related to the present study. Section 3 describes the research methodology with data description and methods used in this study along with the algorithm of proposed model. Section 4 contains the proposed experimental setup. Section 5 represents the predicted results graphically as well as the classification metric along with a comparative analysis of the proposed model. Section 6 contains the discussion of various Covid-19 affected educational parameters along with Covid-19 and human body cell interaction. Section 7 gives the conclusion and future work of the study.

\section{Literature review}

The current age of artificial intelligence and data science allows us to learn everything online from our homes. It is indispensable to design quality content with every possible 
demonstration as seen in the real scenario. On the other hand, it is compulsory to provide better internet connectivity to remote areas so that students cannot suffer from their educational perspective. In this section, we summarize the most recent related work from a review of the literature.

Machine and deep learning algorithms are applied to predict Covid-19 cases for Brazil. Several methodologies were applied among which the support vector regression (SVR) method derives the best results. An error range of 0.87 to 6.90 has been evaluated while forecasting number of Covid-19 cases [5]. Chest X-rays of Covid-19 are taken into consideration to detect the pulmonary manifestation with an iteratively pruned deep learning ensemble model is designed on openly available CXR collections and achieved an accuracy of $99.01 \%$ and area under the curve as 0.9972 [6]. Long short term memory (LSTM), Xgboost, and K-means algorithms are being used to design a model for the short term prediction of Covid-19 cases in Louisana state of USA. K-means-LSTM model outperforms other model combinations and predicts the cases with 601.20 RMSE value [7]. Different models have been proposed based on Convolutional Neural Networks, Deep Neural Networks and Long Short Term Memory also a doublestacked DNN model has been created which is highlighted as the best predictive models. Covid-19 confirmed cases have been predicted for China and Tunisia which achieved an accuracy of $99.97 \%$ and having RMSE of $2.47 \%$. [8]. A spatiotemporal epidemic model based Convolutional LSTM ensemble is proposed to forecast the Covid-19 cases in USA and Italy. The experimentation is focused on multiple external features that might be responsible for spread of Covid-19 throughout the world. One more effort have been incepted by the authors to combine the different ensemble models of different countries into one single model so that models can be trained through transfer learning and help predicting cases with more accuracy [9]. Covid-19 patient education materials have been accessed by everyone easily and are the quintessential way to explore such knowledge. Therefore, it is important to flow genuine and correct knowledge of disastrous pandemic which intern helps in its mitigation. The readability of the content has been analyzed using simple measure and gunning frequency of Gobbledygook and Flesh-Kincaid grade level indices. PEMAT-P has scored good understandability and poor actionability for the same content [10]. The clinical reports are labeled into four classes namely SARS, Covid, ARDS and both Covid, ARDS and are extracted from 212 clinical reports. Classification has been done on four different classes using machine learning algorithms to extract the features of Covid-19. Multinomial Naive Bayesian classifier and regression performs better than other classification models and achieved an accuracy of $96.20 \%$. Authors also classified the disease based on gender implying that weather males are getting affected more than females or vice versa [11]. The prediction models have been investigated using artificial intelligence in order to mitigate the Covid-19 mortality rate. Numerous performance metrics are used to find the competence of the proposed models. Auto seasonal auto regressive integrated moving average model overcomes the performance of other models [12]. To detect the hotspots of the Covid-19 in India, four factors are taken into considerations which are foreign tourist's arrival in India, total population, reported confirmed cases and population density. According to GetisOrd $\mathrm{Gi}^{*}$ statistics it is found that total population and reported confirmed cases are mainly responsible for the hotspots with greater z-score value [13]. Spatial and non-spatial regression models were applied to find out the geographical relevance of the potential factors that are spreading the transmission of the Covid-19 in Bangladesh. Spatial auto-correlation and regression analysis shows that the influential reason for the spread of Covid-19 transmission on such huge rate was population density [14]. Machine learning algorithms are used to analyze the issues related to Covid-19 in India. Total number of confirmed and recovered cases has been predicated using linear regression model. Linear and exponential growth has been seen in the number of Covid-19 confirmed and recovered cases in India [15].

\section{Research methodology}

\subsection{Data description}

In this study, the dataset of Covid-19 has been taken from the official website of the World Health Organization (WHO) [1]. Confirmed and death cases for experimentation are considered from the day when the first Covid-19 cases are registered within the country. The rise in Covid-19 cases has been shown graphically in Fig. 1 and Fig. 2 as of 26th April 2021.

Using MinMaxScaler we have normalized our raw data and make it suitable to compare the statistics and visualize the Covid-19 confirmed and death cases, a scikit-learn library of python is used for this purpose. The raw data we have collected is inconsistent in nature, so the implementation of normalization of the data becomes important. The original form of the data can't be visualized graphically, so to make its clear visibility we scale our data within some range. Datasets used in this study to carry out experimental analysis with the portioning of data as shown in Table 2 . 


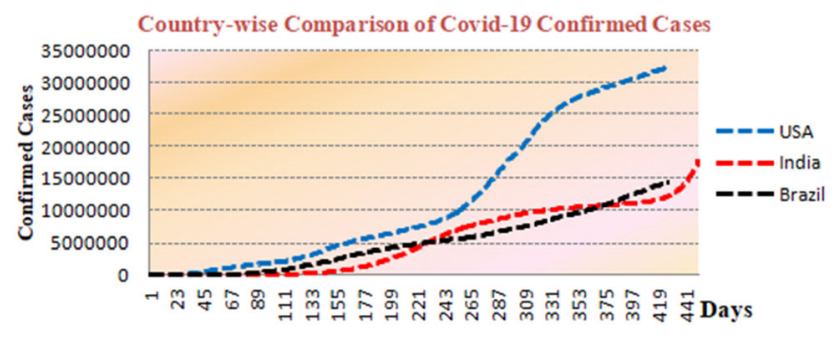

Fig. 1 Covid-19 Actual Confirmed Cases of USA, India, and Brazil

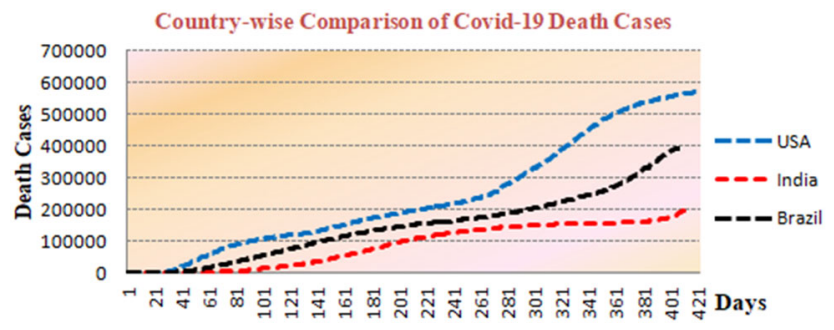

Fig. 2 Covid-19 Actual Death Cases of USA, India, and Brazil

\subsection{Methods}

\subsubsection{LSTM network}

Deep neural networks are a special kind of neural network with deep structural learning networks and are better than traditional neural networks. A recurrent neural network (RNN) is fairly a deep neural network that can find temporal correlations in historical time series predictive system and can sustain the information from previous events. The looping of combinational networks can allow the RNNs to capture the information hidden within the network. RNNs looping networks can take input and raw knowledge from the earlier networks, process the information, and give better predictability about the results to the networks next to it [16]. RNNs are not capable of learning long-term dependencies from the historical time series data but it still works better than exponential smoothing and autoregressive integrated moving average models [17]. Because of its incompetency to model long-term dependencies there exists the vanishing and exploding gradient problems in RNN which leads to the inception of Long Short Term Memory (LSTM) to overcome these limitations. LSTM is a variant of RNN which add the memory cells into it and make it capable of learning long term dependencies [18]. The hidden patterns of historical time series data can be learned by the LSTM to get better future predictability. LSTM is working with three basic gates called input gate $\left(i_{t}\right)$, Output gate $\left(O_{t}\right)$, and Forget gate $\left(f_{t}\right)$. Where, $x_{t}$ is the current input, $c_{t-1}$ is memory cell state, and $h_{t}$ is the current output. The LSTM working steps are shown mathematically in the following Eqs. (1-5).

$i_{t}=\sigma\left(W_{x i} x_{t}+W_{h i} h_{t-1}+W_{c i} c_{t-1}+b_{i}\right)$

$f_{t}=\sigma\left(W_{x f} x_{t}+W_{h f} h_{t-1}+W_{c f} c_{t-1}+b_{f}\right)$

$c_{t}=f_{t} c_{t-1}+i_{t} \tanh \left(W_{x c} x_{t}+W_{h c} h_{t-1}+b_{c}\right)$

$o_{t}=\sigma\left(W_{x o} x_{t}+W_{h o} h_{t-1}+W_{c o} c_{t}+b_{o}\right)$

$h_{t}=o_{t} \tanh \left(c_{t}\right)$

Here $i, f, c$, and $o$ are input gate, forget gate, memory cell, and output gate respectively. Equation (1) is to decide what information should be added bypassing $h_{t-1}$ and $x_{t}$ through the sigmoid layer. In Eq. (2), it says if the relevant information from the prior states has vanished with some probability function or not, here $W_{x, c . f}$ are weight matrices with $b_{f}$ as the offset and $\sigma$ is the logistic sigmoid function. In Eq. (3), $h_{t-1}$ and $x_{t}$ with some weight metric are passed through the tanh layer to get some new information. Equation (4) shows the LSTM output gate with the inclusion of $x_{t}, h_{t-1}$, and $c_{t}$ with some weight metric and sigmoid function applied on. The final output is shown in Eq. (5) with some information passed through tanh layer and multiplied with output gate, and $W_{x i, f, c, o}$ are weight matrices from the memory cell to gate units used in other equations [19].
Table 2 Data selected for experimentation

\begin{tabular}{lll}
\hline Country & Confirmed cases & Death cases \\
\hline USA & 7th Feb. 2020-26th April 2021 & 29th Feb. 2020-26th April 2021 \\
India & 30th Jan. 2020-26th April 2021 & 12th Mar. 2020-26th April 2021 \\
Brazil & 26th Feb. 2020-26th April 2021 & 17th Mar. 2020-26th April 2021 \\
\hline
\end{tabular}




\subsubsection{Bi-directional LSTM}

Bi-directional Long Short Term Memory (BD-LSTM) is the extension to the traditional RNNs which can work in both directions (forward and backward). BD-LSTM can be trained using all prior information available as input and for the specific future time frame. Division of RNNs cell state neuron into positive and negative time frames are responsible for the forward and backward moment of BDLSTM. Backward state outputs are not used as input for the forward states and vice-versa [20]. Using both states for BD-LSTM is compulsory to use to hold its property if in any case backward states are removed it then works like a unidirectional forward recurrent neural network and if forward states are removed it works like the reversed axis of time for regular RNN. We can iteratively compute the hidden sequences for time $t=1$ tot in case of backward states. Mathematically, BD-LSTM can be shown for layer $L$ and time $t$ as in Eqs. (6-12) [21].

$\overleftarrow{f}_{t}^{L}=\sigma\left(W_{\overleftarrow{f} h}^{L} h_{t+1}^{L}+W_{\overleftarrow{f} h}^{L} h_{t}^{L-1}+b_{\overleftarrow{f}}^{L}\right)$

$\overleftarrow{i}_{t}^{L}=\sigma\left(W_{\overleftarrow{i} h}^{L} h_{t+1}^{L}+W_{\overleftarrow{i x}}^{L} h_{t}^{L+1}+b_{\overleftarrow{i}}^{L}\right)$

$\overleftarrow{\tilde{c}}_{t}^{L}=\tanh \left(W_{\overleftarrow{\tilde{c}} h}^{L} h_{t+1}^{L}+W_{\overleftarrow{\tilde{c}} x}^{L} h_{t}^{L-1}+b \underset{\tilde{c}}{L}\right)$

$\overleftarrow{c}_{t}^{L}=\overleftarrow{f}_{t}^{L} \cdot \overleftarrow{c}_{t+1}^{L}+\overleftarrow{i}_{t}^{L} \cdot \overleftarrow{\tilde{c}}_{t}^{L}$

$\overleftarrow{o}_{t}^{L}=\sigma\left(W_{\overleftarrow{o} h}^{L} h_{t+1}^{L}+W_{\overleftarrow{o} x}^{L} h_{t}^{L-1}+b_{\overleftarrow{o}}^{L}\right)$

$\overleftarrow{h}_{t+1}^{L}=\overleftarrow{o}_{t}^{L} \cdot \tanh \left(\overleftarrow{c}_{t}^{L}\right)$

The Summation of the forward layer and backward layer outputs such as $\vec{h}_{t+1}$ and $\overleftarrow{h}_{t+1}$ respectively will result in the output of Bi-directional LSTM.

$y_{t}=W_{\overrightarrow{h y}} \vec{h}_{t+1}+W_{\vec{h}_{y}} \overleftarrow{h}_{t+1}+b_{y}$

$f_{t}$ is forget gate, $i_{t}$ is input gate, $O_{t}$ is Output gate, $c_{t}$ is memory cell state, $h_{t}$ is the current output and $y_{t}$ is the combination of current state values in both directions. $\mathrm{W}_{\mathrm{f}}$, $\mathrm{W}_{\mathrm{i}}, \mathrm{W}_{\mathrm{o}}$, and $\mathrm{W}_{\mathrm{c}}$ are the weight matrices and $\mathrm{b}_{\mathrm{f}}, \mathrm{b}_{\mathrm{i}}, \mathrm{b}_{\mathrm{o}}, \mathrm{b}_{\mathrm{c}}$ are four bias vectors. To determine outputs for the forward pass, BD-LSTM inputs need to be processed for $1 \leq t \leq T$ time. To ensure both states, execution of both forward and backward passes for time $t=1$ toT and $t=T$ to 1 is required respectively. Furthermore, a forward pass can be executed to determine the output of neurons. For the backward pass, the objective function derivative is calculated for the same time as processed for the forward pass. Then, output neurons will be ensured by evaluating backward pass again, and forward states are ensured for $t=T t o 1$ time when the backward pass is executed. Lastly, weights are needed to upgrade.

\subsubsection{Convolutional LSTM}

Convolutional Long Short Term Memory (ConvLSTM) is an ensemble of CNN and LSTM which collectively gives one strong model (ConvLSTM) to learn and extract the number of features that one can't extract with human intervention and experience. CNN and LSTM both are deep neural networks, the combination of which also gives the strong deep neural network as ConvLSTM [22]. The limitation of the LSTM network is that it can process the one-dimensional data by keeping the temporal dependencies only. LSTM can't process the spatial dependencies at all like the fully connected LSTM networks. Unlike the matrix multiplication in LSTM, ConvLSTM uses the Convolutional operation in both states to state and input to state transitions. ConvLSTM also evaluates the future state of the cell using current input and past states of its neighbors [23]. Mathematically, ConvLSTM can be represented as in Eqs. (13-17). Here, $(*)$ is the Convolutional operator between states and from input to state transitions, and '.' represents Hadamard product.

$i_{t}=\sigma\left(W_{x i} \times x_{t}+W_{h i} \times h_{t-1}+W_{c i} \cdot c_{t-1}+b_{i}\right)$

$f_{t}=\sigma\left(W_{x f} \times x_{t}+W_{h f} \times h_{t-1}+W_{c f} \cdot c_{t-1}+b_{f}\right)$

$c_{t}=f_{t} \cdot c_{t-1}+i_{t} \cdot \tanh \left(W_{x c} \times x_{t}+W_{h c} * h_{t-1}+b_{c}\right)$

$o_{t}=\sigma\left(W_{x o} \times x_{t}+W_{h o} \times h_{t-1}+W_{c o} \cdot c_{t}+b_{o}\right)$

$h_{t}=o_{t} \cdot \tanh \left(c_{t}\right)$

Here $h_{1}, h_{2} \ldots h_{t}$ are hidden states, $x_{1}, x_{2}, \ldots x_{t}$ are inputs, $c_{1}, c_{2} \ldots c_{t}$ are cell outputs and $i_{t}, o_{t}, f_{t}$ are different gates [21]. $f_{t}$ is forget gate, $i_{t}$ is input gate, $O_{t}$ is Output gate, $c_{t}$ is memory cell state, $h_{t}$ is the current output and $y_{t}$ is the combination of current state values in both directions. $\mathrm{W}_{\mathrm{f}}$, $\mathrm{W}_{\mathrm{i}}, \mathrm{W}_{\mathrm{o}}$, and $\mathrm{W}_{\mathrm{c}}$ are the weight matrices and $\mathrm{b}_{\mathrm{f}}, \mathrm{b}_{\mathrm{i}}, \mathrm{b}_{\mathrm{o}}, \mathrm{b}_{\mathrm{c}}$ are four bias vectors. CNN activations can produce the feature maps which intern can be used as input to the LSTM network. Therefore, ConvLSTM can handle both temporal and spatial operations.

$i_{t}$ is to decide what information should be added bypassing $h_{t-1}$ and $x_{t}$ through the sigmoid layer. $f_{t}$ is used when relevant information from the prior states has vanished with some probability function or not, here $W_{x, c . f}$ are weight matrices with $b_{f}$ as the offset and $\sigma$ is the logistic sigmoid function. $c_{t}$ includes $h_{t-1}$ and $x_{t}$ with some weight metric are passed through the tanh layer to get some new information. $o_{t}$ shows the LSTM output gate with the inclusion of $x_{t}, h_{t-1}$, and $c_{t}$ with some weight metric and sigmoid function applied on. The final output is shown in $h_{t}$ 
with some information passed through tanh layer and multiplied with output gate, and $W_{x i, f, c, o}$ are weight matrices from the memory cell to gate units used.

\subsection{Algorithm}

Input: Raw Covid-19 Dataset $\left(\mathrm{CD}_{\mathrm{R}}\right)$.

Output: Best model $\left(\mathrm{M}_{1}, \mathrm{M}_{2}, \mathrm{M}_{3}\right)$.

Algorithms used: Bi-directional LSTM (), Convolutional LSTM (), CoBiD ensemble ().

Output: Best model $\left(\mathrm{M}_{1}, \mathrm{M}_{2}, \mathrm{M}_{3}\right)$

1. $\mathrm{CD}_{\mathrm{P}}=$ Data_Preprocessing $\left(\mathrm{CD}_{\mathrm{R}}\right)$

2. Data_Splitting \{Training_Data + Test_Data $=$ $\left.\mathrm{CD}_{\mathrm{P}}\right\}$

3. Apply Data_Splitting on $\mathrm{CD}_{\mathrm{P}}$

4. If $\mathrm{T}_{\mathrm{d}}=0.75$ than $\mathrm{T}_{\mathrm{t}}=\mathrm{CD}_{\mathrm{P}}-0.75$

5. $\mathrm{M}_{\mathrm{T}(\mathrm{E} 1)}=$ Bi-directional LSTM $\left(\mathrm{T}_{\mathrm{d}}\right)$

6. $\mathrm{M}_{\mathrm{T}(\mathrm{E} 2)}=$ Convolutional LSTM $\left(\mathrm{T}_{\mathrm{d}}\right)$

7. $\mathrm{CoBiD}$ ensemble $=$ Bi-directional LSTM + Convolutional LSTM

8. $\left.\mathrm{M}_{\mathrm{T}(\mathrm{E}}{ }^{1}\right)=$ CoBiD ensemble $\left(\mathrm{T}_{\mathrm{d}}\right)$

9. $\mathrm{M}_{1}=\mathrm{M}_{\mathrm{T}(\mathrm{E} 1)}\left(\mathrm{T}_{\mathrm{t}}\right)$

10. $\mathrm{M}_{2}=\mathrm{M}_{\mathrm{T}(\mathrm{E} 2)}\left(\mathrm{T}_{\mathrm{t}}\right)$

11. $\mathrm{M}_{3}=\mathrm{M}_{\mathrm{T}(\mathrm{E})}^{1}\left(\mathrm{~T}_{\mathrm{t}}\right)$

12. Compare_Accuracy $\left(\mathrm{M}_{1}, \mathrm{M}_{2}, \mathrm{M}_{3}\right)$

\section{Experimental setup}

The experimentation is carried out with open source libraries like Pandas, PyTorch, Matplotlib, Numpy, Keras, Tensorflow, and Pandas using Python 3.3 in Google Colaboratory. The experimental setup is based on a working environment having Intel(R) Core (TM) i5-7400 CPU @ 3.00 GHz with 4 GB RAM under 64-bit Windows

10 Pro Operating system. In this paper, Covid-19 confirmed and death cases of USA, India, and Brazil have been forecasted using three different experimental setups. (i) The experiment performed using Bi-directional LSTM. (ii) The experiment performed using Convolutional LSTM. (iii) The experiment is performed using an ensemble of $\mathrm{Bi}$ directional and Convolutional LSTM known as the CoBiDNet ensemble. Covid-19 confirmed and death cases are forecasted for the next 70 days with each of the experiment and the visualization of results are shown graphically in Sect. 5. Dataset is categorized into training and testing sets with a proportion of $75 \%$ and $25 \%$ respectively. Different classification metrics like accuracy, precision, recall, f-measure, and mean absolute percentage error (MAPE) are used to find the veracity of the proposed model and are shown in Tables 3 and 4. The different experimental setups are discussed below.

For Bi-directional LSTM, the input is Covid-19 confirmed and death cases in.csv format along with necessary io imports. Pandas library functions like Pandas.dataframe.sum() is used for axis zero and to handle the missing values within the input data. Pandas.to_datetime() is used to convert the argument string date time into python date-time object. Furthermore, after refinement of data, we split our input data into training and testing parts, and by importing the scikit-learn MinMaxScaler() we transform the training data into a specified range and normalized it for better use. Numpy.reshape() is used to reshape the data according to the model need without any change within the array data. Then, we apply the Bi-directional LSTM model with ReLu activation function to overcome the problem of vanishing gradient. The non-linearity property is added by the inclusion of the dense layer. Adam optimizer is used, which is an optimization algorithm to update the weights of the network, and mean square error is used as a loss function to evaluate the model. Model is trained for 500

Table 3 Classification metrics on covid-19 confirmed cases of USA, India, and Brazil

\begin{tabular}{|c|c|c|c|c|c|c|}
\hline S. No & Models & Accuracy & Precision & Recall & F-measure & MAPE \\
\hline \multicolumn{7}{|l|}{$U S A$} \\
\hline 1 & BD-LSTM & 95.80 & 100 & 80.71 & 0.90 & 4.20 \\
\hline 2 & ConvLSTM & 96.87 & 100 & 84.81 & 0.90 & 3.13 \\
\hline 3 & CoBiD-Net & 99.09 & 100 & 90.00 & 0.94 & 0.91 \\
\hline \multicolumn{7}{|l|}{ India } \\
\hline 1 & BD-LSTM & 96.77 & 100 & 91.66 & 0.95 & 3.23 \\
\hline 2 & ConvLSTM & 97.80 & 100 & 91.79 & 0.95 & 2.20 \\
\hline 3 & CoBiD-Net & 99.10 & 100 & 98.98 & 0.99 & 0.90 \\
\hline \multicolumn{7}{|l|}{ Brazil } \\
\hline 1 & BD-LSTM & 97.70 & 100 & 84.00 & 0.88 & 2.30 \\
\hline 2 & ConvLSTM & 98.74 & 100 & 91.90 & 0.95 & 1.26 \\
\hline 3 & CoBiD-Net & 99.13 & 100 & 95.73 & 0.97 & 0.87 \\
\hline
\end{tabular}


Table 4 Classification metrics on covid-19 death cases of USA, India, and Brazil

\begin{tabular}{|c|c|c|c|c|c|c|}
\hline \multicolumn{7}{|c|}{ Classification Metrics on Covid-19 Death Cases } \\
\hline S.No & Models & Accuracy & Precision & Recall & F-measure & MAPE \\
\hline \multicolumn{7}{|l|}{ USA } \\
\hline 1 & BD-LSTM & 95.00 & 100 & 80.00 & 0.82 & 5.00 \\
\hline 2 & ConvLSTM & 96.87 & 100 & 87.61 & 0.91 & 3.13 \\
\hline 3 & CoBiD-Net & 98.40 & 100 & 85.35 & 0.90 & 1.60 \\
\hline \multicolumn{7}{|l|}{ India } \\
\hline 1 & BD-LSTM & 95.00 & 100 & 85.00 & 0.85 & 5.00 \\
\hline 2 & ConvLSTM & 95.80 & 100 & 89.61 & 0.91 & 4.20 \\
\hline 3 & CoBiD-Net & 98.10 & 100 & 92.05 & 0.90 & 1.90 \\
\hline \multicolumn{7}{|l|}{ Brazil } \\
\hline 1 & BD-LSTM & 94.00 & 100 & 86.92 & 0.86 & 6.00 \\
\hline 2 & ConvLSTM & 96.87 & 100 & 89.71 & 0.92 & 3.13 \\
\hline 3 & CoBiD-Net & 98.30 & 100 & 95.72 & 0.96 & 1.70 \\
\hline
\end{tabular}

epochs with having validation split as 0.2 and verbose equals to 1 . Testing is performed on the trained model and the results are evaluated in terms of accuracy, precision, recall, f-measure, and MAPE, also at last the forecasted cases are visualized graphically.

For Convolutional LSTM, the input is the same as in the BD-LSTM, and Pandas and Numpy libraries used are also the same as the previous experimental setup. Here also the trained dataset is normalized using MinMaxScaler(). Split_sequence() function is used to reshape the input data for the model. In ConvLSTM input shape of the training data is (samples, time_steps, rows, columns, features). The one-dimensional copy of the array is returned using flatten() and also dense layer is used to hold non-linearity. Adam optimizer is used for weight updating along with mean square error to calculate model efficiency. Model is trained for 500 epochs with verbose equals 1 and validation split as 0.2 .

For the proposed CoBiD-Net ensemble as shown in Fig. 3, the input dataset is in.csv format followed by Pandas.dataframe.sum() to handle missing values. CoBiD-Net ensemble is the synergy of Convolutional and $\mathrm{Bi}$ -
Fig. 3 Proposed CoBiD-Net ensemble framework

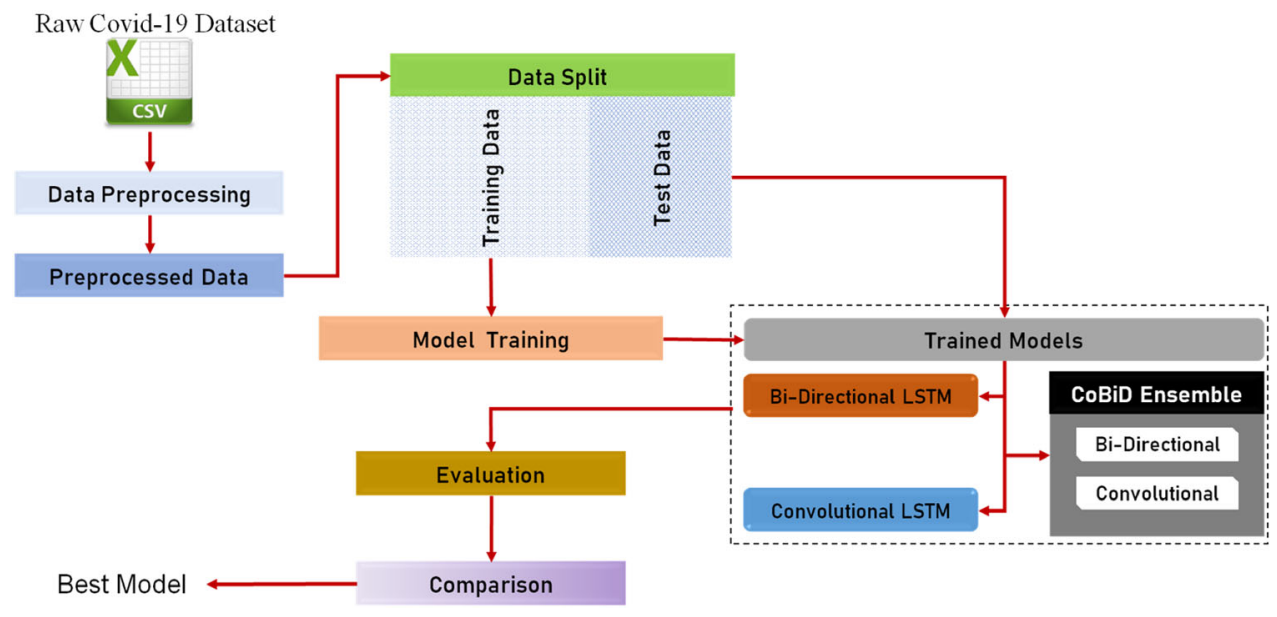



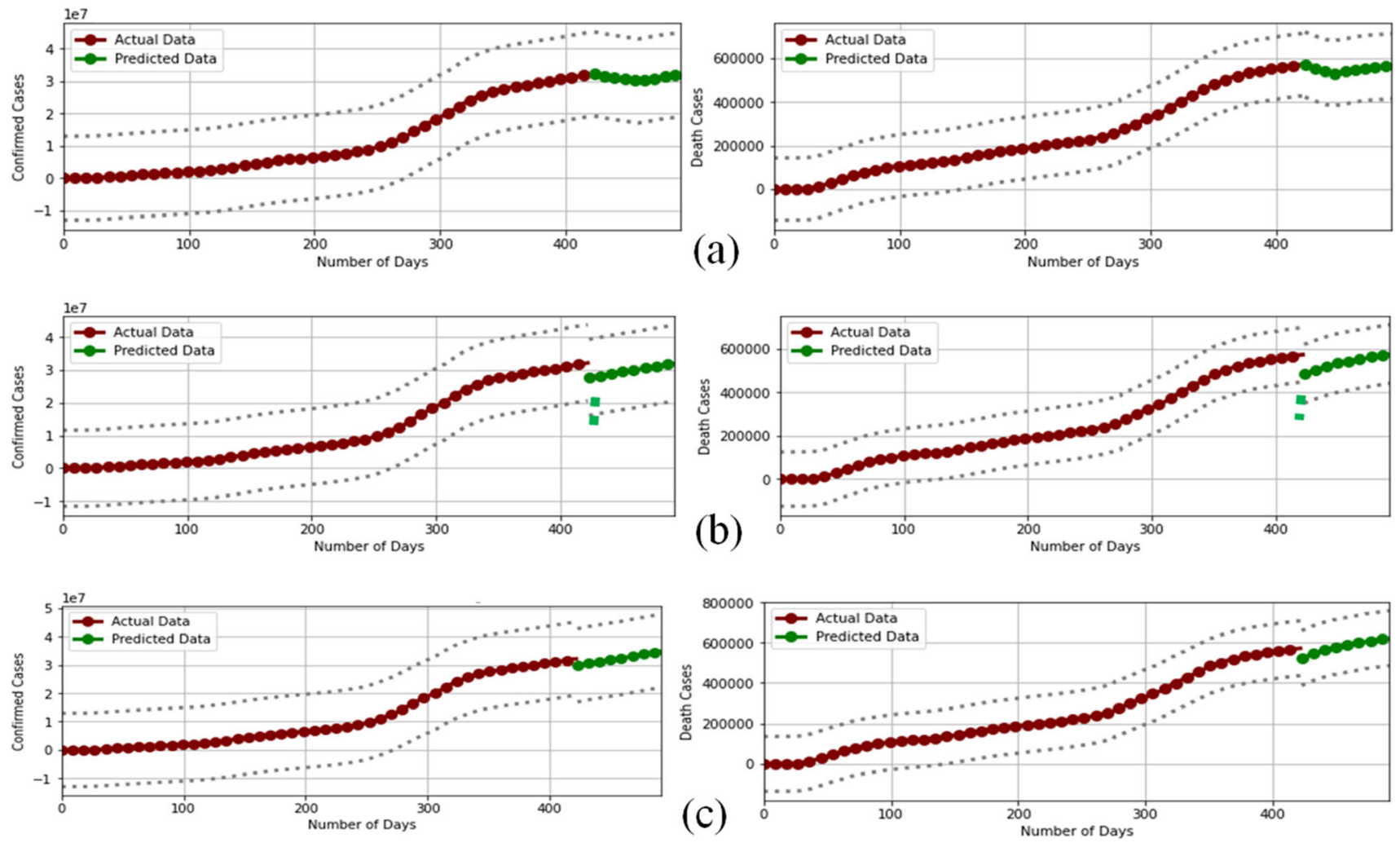

Fig. 4 USA Covid-19 confirmed and death cases forecasting a using Bi-directional LSTM b using Convolutional LSTM c using CoBiD-Net Ensemble

directional LSTM networks. After handling missing values we use Pandas.to_datetime() to convert string date time to python date-time object. MinMaxScaler is used to map the input values into the specified range and normalize the data. Here, split_sequence() function is used to reshape the data for the proposed model. StratifiedKFold is imported from the sklearn library used for K-fold cross-validation and it shuffles the data before splitting is done. ConvLSTM2D with 64 filters and kernel size as $(1,2)$ is used along with ReLu activation function to avoid vanishing gradient problem. Flatten() and a dense layer is used followed by Adam optimizer and mean square loss function. Bi-directional LSTM is added with 100 hidden units along with ReLu activation function. For BD-LSTM also flatten() is used along with dense layer. Model.LinearRegression() is used to compile the results of both models into one model. Hence, test data is tested on this model to evaluate the classification metrics, and also the forecasted results are visualized graphically.

\section{Results and comparative analysis}

A recurrent neural network (RNN) based Long Short Term Memory (LSTM) can be used to find the temporal correlations in historical time series and can retain the information from previous events. This feature can help the LSTM models to learn better from the historical data and predict the future events. This is the sole reason why LSTM models are being used in this study to find the forecasted results in case of Covid-19 confirmed and death cases.

\subsection{Impact of Covid-19 on USA}

Bi-directional LSTM, Convolutional LSTM and CoBiDNet Ensemble is used to forecast the number of Covid-19 confirmed and death cases for the USA as shown in Fig. 4. Actual Covid-19 confirmed and death cases are shown in dark red line and predicted Covid-19 confirmed and death cases are shown in green color. The significant decrease in the number of predicted cases has been shown and then again in future cases will rise. 

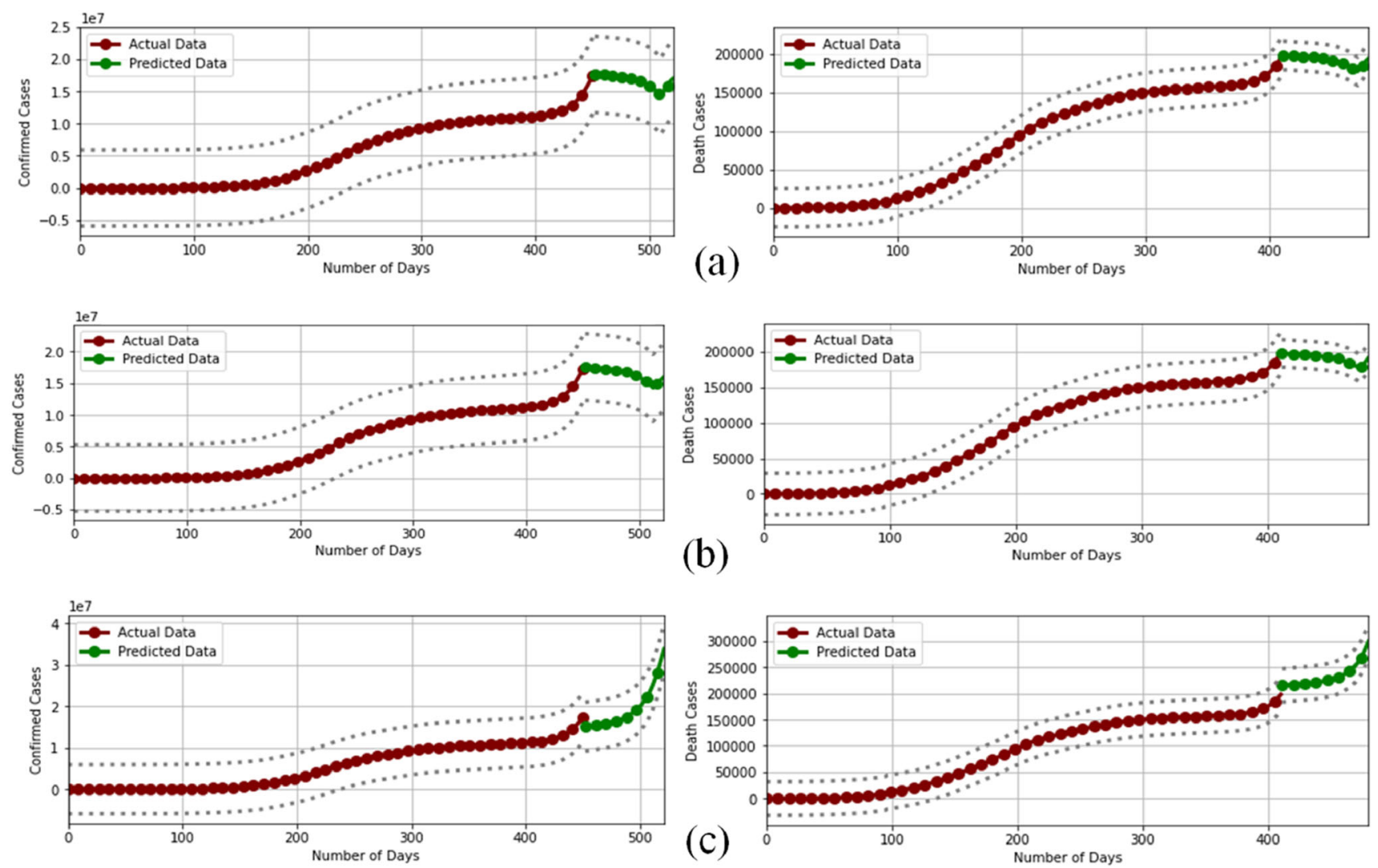

Fig. 5 India Covid-19 confirmed and death cases forecasting a using Bi-directional LSTM b using Convolutional LSTM c using CoBiD-Net Ensemble

\subsection{Impact of Covid-19 on India}

Bi-directional LSTM, Convolutional LSTM and CoBiDNet Ensemble is used to predict the number of Covid-19 confirmed and death cases for India as shown in Fig. 5. Actual Covid-19 confirmed and death cases are shown in dark red line and predicted Covid-19 confirmed and death cases are shown in green color.

\subsection{Impact of Covid-19 on Brazil}

Bi-directional LSTM, Convolutional LSTM and CoBiDNet Ensemble is used to predict the number of Covid-19 confirmed and death cases for Brazil as shown in Fig. 6. The actual and forecasted cases are shown in different colors to easily draw the attention on increasing Covid-19 cases in future also. The decrease in the number of predicted cases has been shown for the short period and then again in future cases will rise.

Section 5.1 to 5.3 shows the Covid-19 confirmed and death cases prediction for the USA, India, and Brazil using three deep learning approaches. Actual (dark red line) and forecasted (green line) Covid-19 confirmed and death cases data has been shown graphically from Fig. 4 to Fig. 6 .
Covid-19 confirmed and death cases have been forecasted for the next 70 days in which all the three proposed models show the decrease in cases for the first couple of weeks and then again cases will be raised up. The proposed CoBiDNet ensemble model shows the sudden decrease in forecasted Covid-19 confirmed and death cases for the near future. Again Covid-19 confirmed and mortality cases will be increased after a significant drop in the number of cases. This may act as a trap for the government and the common man as everyone thinks that Covid-19 is about to end now, which may not be true for the time to come. Studies also revealed the hike in Covid-19 cases because of a new strain of Covid-19 in some countries.

\subsection{Comparative analysis}

A comparative analysis of three experimental setups proposed in this paper with the already published work is shown in Table 5. Our proposed CoBiD-Net ensemble shows greater accuracy with the minimum mean absolute percentage error than the literature reviewed in the comparative analysis. 

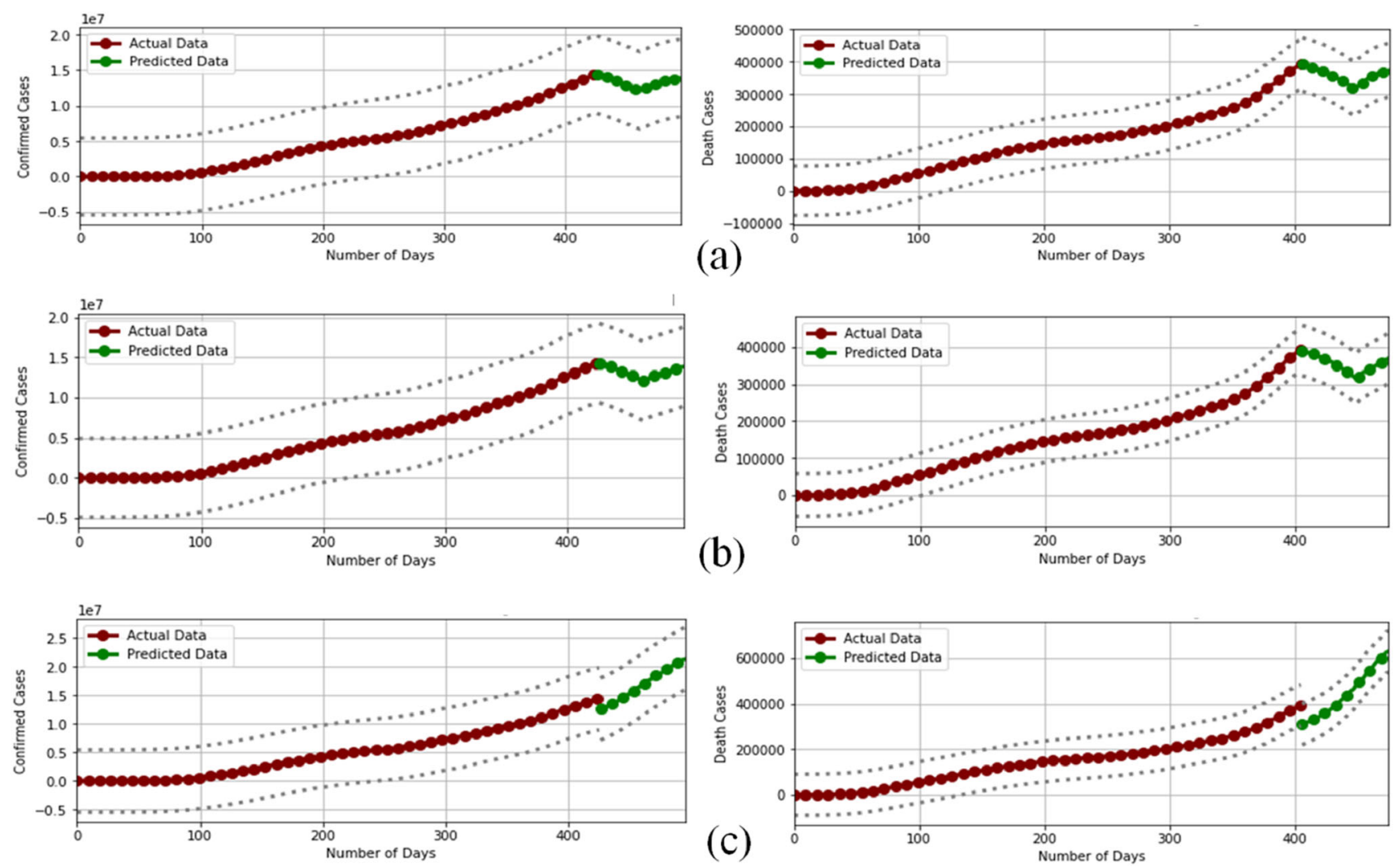

Fig. 6 Brazil Covid-19 confirmed and death cases forecasting a using Bi-directional LSTM b using Convolutional LSTM $\mathbf{c}$ using CoBiD-Net Ensemble

\section{Discussion}

The World Health Organization (WHO) has declared the pandemic of the novel SARS-CoV2 infection early last year which became a major public health challenge worldwide. The spread of COVID-19 has sent shockwaves throughout the world. By January 12, 2021, 9 billion cases of COVID-19 worldwide had been confirmed and COVID-19-related deaths were more than 1.9 million [30]. The castrating and extreme impacts of COVID-19 have petrified the world to its core. The COVID-19 pandemic is eccentric in terms of its virulence, the continuing risks associated with the asymptomatic carriers who may still spread the virus at a high rate, the impacts of the proprietary on population, the probability of second or third waves, and the recognition \& attention it has gained due to its global presence. The endothelial dysfunction of epithelial cells and Angiotensin-Converting Enzyme 2 receptor (ACE2) with the Covid-19 is shown architecturally in Fig. 7.

\subsection{COVID-19 and institutional closures}

According to World Vision, "100 million children and young people are affected by natural disasters every year and most of them face disruption to their schooling." Likewise, the COVID-19 pandemic has affected educational programs globally, contributing to the nearly complete closing of schools, universities, and colleges. As of 30 September 2020, approximately 1.077 billion learners were badly influenced due to school closures in response to the pandemic and according to UNICEF monitoring, 53 countries are currently implementing nationwide closures (mostly in Latin America, Africa \& Canada) and 27 are implementing local closures (Portugal, Spain, and India) as an attempt to control or delay the spread that affects about 61.6 percent of the global student population. The Indian union government announced a countrywide lock-down of schools and colleges on March 16, 2020. Educational institutions in 72 countries' such as Greenland, USA, China, Norway, and France are currently open [31]. There were few local outbreaks where institutions were opened after a partial closure. One such incident occurred in the United States where residence halls were opened on August 17, and there were over 700 infected coronavirus cases among the students within two months. Furthermore, the pandemic implications include the possible use of school facilities as makeshift hospitals and quarantine centers such as done with $\sim 600$ universities \& colleges in India. This might 
Table 5 Comparative analysis of the proposed CoBiD-Net ensemble model with previous benchmark studies

\begin{tabular}{|c|c|c|c|}
\hline Model used & Dataset used & Motive & Measures \\
\hline $\begin{array}{l}\text { Multi-head attention, } \\
\text { LSTM, CNN [24] }\end{array}$ & $\begin{array}{l}\text { Covid-19 data from John } \\
\text { Hopkins University }\end{array}$ & Short term and long term forecasting & $\begin{array}{l}\text { SMAPE of } 0.25 \text { for short term and } 2.59 \text { for } \\
\text { long term forecasting }\end{array}$ \\
\hline RNN based LSTM [25] & Covid-19 India & Forecast the number of cases & $\begin{array}{l}\text { Test and train LSTM MSE of } 0.59 \text { and } 0.26 \\
\text { respectively }\end{array}$ \\
\hline $\begin{array}{l}\text { Deep LSTM ensemble } \\
\text { model [26] }\end{array}$ & Covid-19 India & $\begin{array}{l}\text { Forecast the number of confirmed } \\
\text { and death cases }\end{array}$ & $\begin{array}{l}\text { Accuracy of } 97.59 \text { and } 98.88 \text { for confirmed } \\
\text { and death cases respectively }\end{array}$ \\
\hline ARIMA, SVR, LSTM [27] & $\begin{array}{l}\text { Top ten Covid-19 affected } \\
\text { countries }\end{array}$ & Forecast the number of cases & MAE of 0.0070 and RMSE of 0.0077 \\
\hline ARIMA, CUBIST, RF [5] & Covid-19 Brazil & $\begin{array}{l}\text { Short term forecasting of confirmed } \\
\text { cases }\end{array}$ & $0.95 \%$ to $6.90 \%$ error for 6 days forecasting \\
\hline $\begin{array}{l}\text { LSTM and curve-fitting } \\
\text { [28] }\end{array}$ & Covid-19 India & Forecast Covid-19 cases for 30 days & Error up to $8 \%$ for the total confirmed cases \\
\hline $\begin{array}{l}\text { Covid-19Net framework } \\
\text { [29] }\end{array}$ & $\begin{array}{l}\text { Covid-19 cases of } \\
\text { Germany, Italy, and Spain }\end{array}$ & $\begin{array}{l}\text { Predict the number of confirmed } \\
\text { cases }\end{array}$ & $\begin{array}{l}\text { MAPE of } 1.44,1.80 \text { and } 2.82 \text { for Germany, } \\
\text { Italy and Spain respectively }\end{array}$ \\
\hline $\begin{array}{l}\text { Experiment using BD- } \\
\text { LSTM in this paper }\end{array}$ & $\begin{array}{l}\text { Covid-19 confirmed and } \\
\text { death cases of USA, India, } \\
\text { and Brazil }\end{array}$ & $\begin{array}{l}\text { Forecast the number of Covid- } 19 \\
\text { confirmed and death cases for the } \\
\text { next } 70 \text { days }\end{array}$ & $\begin{array}{l}\text { MAPE ranges from } 2.30 \text { to } 6 \text { and other } \\
\text { classification metrics are mentioned in } \\
\text { Tables } 3 \text { and } 4\end{array}$ \\
\hline $\begin{array}{l}\text { Experiment using } \\
\text { ConvLSTM in this paper }\end{array}$ & & & $\begin{array}{l}\text { MAPE ranges from } 1.26 \text { to } 4.20 \text { and other } \\
\text { classification metrics are mentioned in } \\
\text { Tables } 3 \text { and } 4\end{array}$ \\
\hline $\begin{array}{l}\text { Experiment using Proposed } \\
\text { CoBiD-Net Ensemble in } \\
\text { this paper }\end{array}$ & & & $\begin{array}{l}\text { MAPE ranges from } 0.87 \text { to } 1.90 \text { and other } \\
\text { classification metrics are mentioned in } \\
\text { Tables } 3 \text { and } 4\end{array}$ \\
\hline
\end{tabular}

lead to protracted disturbance and sustained school instability as shown in Fig. 8 .

\subsection{Recent advances to combat covid-19}

Artificial Intelligence (AI) technologies help to foresee the most-effective public health interventions, accelerating vaccine development, and keep the public updated with scientific developments. Likewise, they have also made it possible for us to move quite a bit of our lives webbased, maintaining educational and financial frameworks when most people are staying home while assisting us to keep ourselves associated with each other. Currently, more than 172 vaccine candidates are in various preclinical and phase 1 to 3 clinical trials using several vaccination strategies including protein-based $(64+)$, nucleic acidbased (39), viral vector-based (37), inactivated or live-attenuated virus-based (15), etc. across the world [33]. Two mRNA-based vaccines; mRNA-1273 \& mRNA BNT162b2 and a non-replicating viral vector-based AZD1222 by AstraZeneca are ready for limited distribution while in India, COVAXIN (inactivated- virus-based vaccine developed by Bharat Biotech) and Covishield (AZD1222) get approval for emergency use [1]. Various global vaccination uptakes have been carried out in many countries, some of them are shown in Table 6. Even after any kind of vaccine availability, to ensure the efficient uptake and global vaccination, we will require behavioral reinforcement so we need to prioritize research that will create the maximum contributions to our understanding of the consequences of, and recovery from, the pandemic.

\section{Conclusion}

The catastrophic phase of Covid-19 has destroyed almost a year for the students due to its unexpected behavior of selfreplication and devastating effect on mankind. There is a sudden shift of the education from face to face interaction to online E-Learning platform with the paralyzed E-Learning infrastructure of most of the countries. Students around the world and in countries like the USA, India, and Brazil get affected due to the Covid-19 outbreak having improper management of learning opportunities. Students from the medical background may suffer due to impractical knowledge of their field, students from engineering and technology background may suffer due to low internet connectivity in remote areas due to which online compilers and debuggers cannot work, and available E-Learning content is also not accessible. So, there is a need to work in 
Fig. 7 Covid-19

Immunopathology
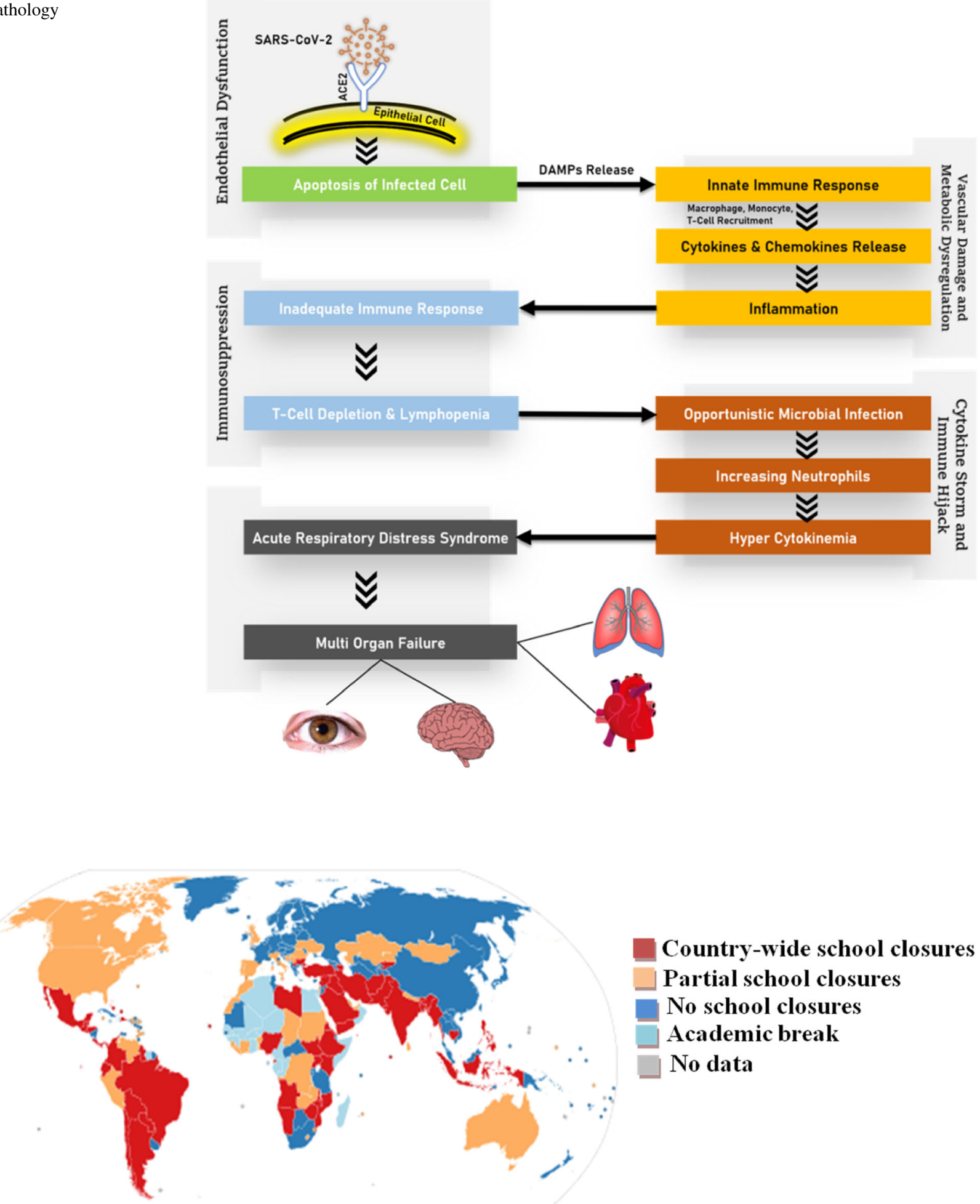

Country-wide school closures

Partial school closures

No school closures

Academic break

No data

Fig. 8 Number of students affected by school closures due to Covid-19 worldwide [32] 


\section{References}

1. "WHO Coronavirus Disease (COVID-19) Dashboard." https:// covid19.who.int/. Accessed 25 April 2021.

2. Dhawan, S. (2020). Online learning: A panacea in the time of COVID-19 Crisis. Journal of Educational Technology Systems, 49(1), 5-22. https://doi.org/10.1177/0047239520934018.

3. Grange, Le., \& L. . (2020). Covid-19 pandemic and the prospects of education in South Africa. Prospects. https://doi.org/10.1007/ s11125-020-09514-w.

4. Singh, P., \& Kaur, R. (2020). An integrated fog and Artificial Intelligence smart health framework to predict and prevent COVID-19. Global Transitions, 2, 283-292. https://doi.org/10. 1016/j.glt.2020.11.002.

5. Ribeiro, M. H. D. M., Silva, R. G., Mariani, V. C., et al. (2020). Short-term forecasting COVID-19 cumulative confirmed cases: Perspectives for Brazil. Chaos, Solitons and Fractals, 135, 109853. https://doi.org/10.1016/j.chaos.2020.109853.

6. Rajaraman, S., Siegelman, J., Folio, L. S., et al. (2020). Iteratively pruned deep learning ensembles for COVID-19 detection in chest X-rays. IEEE Access, 8, 115041-115050.

7. Vadyala, S.R., Betgeri, S.N., Sherer, E., et al. (2020). Prediction of the number of COVID-19 confirmed cases based on K-MeansLSTM. Populations and Evolution. https://doi.org/10.2196/pre prints.20798.

8. Yahia, N. B., Kandara, M. D., Saoud, N. B. B. (2020). Deep ensemble learning method to forecast COVID-19 outbreak. Research Square. https://doi.org/10.21203/rs.3.rs-27216/v1.

9. Paul, S. K., Jana, S., \& Bhaumik, P. (2020). A multivariate spatiotemporal model of COVID-19 epidemic using ensemble of ConvLSTM networks. Journal of The Institution of Engineers (India). https://doi.org/10.1007/s40031-020-00517-x.

10. Kruse, J., Toledo, P., Belton, B. T., et al. (2020). Readability, content, and quality of COVID-19 patient education materials from academic medical centers in the United States. American Journal of Infection Control, 23-26. https://doi.org/10.1016/j. ajic.2020.11.023.

11. Khanday, A. M. U. D., Rabani, S. T., Khan, Q. R., et al. (2020). Machine learning based approaches for detecting COVID-19 using clinical text data. International Journal of Information Technology, 12, 731-739. https://doi.org/10.1007/s41870-020-00495-9.

12. Satpathy, S., Mangla, M., Sharma N., et al. (2021). Predicting mortality rate and associated risks in COVID-19 patients. Spatial Information Research. https://doi.org/10.1007/s41324-021-00379-5.

13. Parvin, F., Ali, S.A., Hashmi S.N.I., et al. (2021). Spatial prediction and mapping of the COVID-19 hotspot in India using geostatistical technique. Spatial Information Research. https:// doi.org/10.1007/s41324-020-00375-1.

14. Sarkar, S.K., Ekram, K.M.M., Das, P.C. (2021). Spatial modeling of COVID-19 transmission in Bangladesh. Spatial Information Research. https://doi.org/10.1007/s41324-021-00387-5.

15. Senapati, A., Nag, A., Mondal, A., et al. (2021). A novel framework for COVID-19 case prediction through piecewise regression in India. International Journal of Information Technology, 13, 41-48. https://doi.org/10.1007/s41870-020-00552-3.

16. Kumar, J., Goomer, R., \& Singh, A. K. (2018). Long short term memory recurrent neural network (LSTM-RNN) based workload forecasting model for cloud datacenters. Procedia Computer Science, 125, 676-682. https://doi.org/10.1016/j.procs.2017.12. 087.

17. Shastri, S., Kour, P., Kumar, S., et al. (2020). A nested stacking ensemble model for predicting districts with high and low maternal mortality ratio (MMR) in India. International Journal of Information Technology. https://doi.org/10.1007/s41870-02000560-3.
18. Gautam, Y. (2021). Transfer Learning for COVID-19 cases and deaths forecast using LSTM network. ISA Trans., no. S0019-0578. https://doi.org/10.1016/j.isatra.2020.12.057.

19. Islam, Z., Islam, M., \& Asraf, A. (2020). A combined deep CNNLSTM network for the detection of novel coronavirus ( COVID19) using X-ray images. Informatics Med. Unlocked, 20, 100412. https://doi.org/10.1016/j.imu.2020.100412.

20. Schuster, M., \& Paliwal, K. K. (2013). Bidirectional recurrent neural network. IEEE Transactions on Signal Processing, 8(2), 1-4. https://doi.org/10.1109/78.650093.

21. Shastri, S., Singh, K., Kumar, S., et al. (2020). Time series forecasting of Covid-19 using deep learning models: India-USA comparative case study. Chaos, Solitons and Fractals, 140, 110227. https://doi.org/10.1016/j.chaos.2020.110227.

22. Shi, X., Chen, Z., \& Wang, H. (2015). Convolutional LSTM network: A machine learning approach for precipitation nowcasting. NIPS'15 Proceedings of the 28th International Conference on Neural Information Processing Systems, 1, 802-810.

23. Nawaz, A., Zhiqiu, H., Senzhang, W., et al. (2020). Convolutional LSTM based transportation mode learning from raw GPS trajectories. IET Intelligent Transport Systems, 14(6), 570-577. https://doi.org/10.1049/iet-its.2019.0017.

24. Abbasimehr, H., \& Paki, R. (2020). Prediction of COVID-19 confirmed cases combining deep learning methods and Bayesian optimization. Chaos, Solitons and Fractals, 110511. https://doi. org/10.1016/j.chaos.2020.110511.

25. Reddy, K. S. S., Reddy, Y. C. A. P., \& Rao, C. M. (2020). Recurrent neural network based prediction of number of COVID19 cases in India. Materials Today Proceedingd, 4-7. https://doi. org/10.1016/j.matpr.2020.11.117.

26. Shastri, S., Singh, K., Kumar, S., et al. (2021). Deep-LSTM ensemble framework to forecast Covid-19: An insight to the global pandemic. International Journal of Information Technology. https://doi.org/10.1007/s41870-020-00571-0.

27. Shahid, F., Zameer, A., \& Muneeb, M. (2020). Predictions for COVID-19 with deep learning models of LSTM, GRU and BiLSTM. Chaos, Solitons and Fractals, 140, 110212. https://doi. org/10.1016/j.chaos.2020.110212.

28. Tomar, A., \& Gupta, N. (2020). Prediction for the spread of COVID-19 in India and effectiveness of preventive measures. Science of the Total Environment, 728, 138762. https://doi.org/ 10.1016/j.scitotenv.2020.138762.

29. Huang, C. J., Shen, Y., Kuo, P. H., et al. (2020). Novel spatiotemporal feature extraction parallel deep neural network for forecasting confirmed cases of coronavirus disease 2019. SocioEconomic Planning Sciences, 100976. https://doi.org/10.1016/j. seps.2020.100976.

30. "Worldometer - real time world statistics." https://www.world ometers.info/. Accessed 18 April 2021.

31. UNESCO, "School closures caused by Coronavirus (Covid-19)," Unesco. 2020. Accessed 18 April 2021. [Online]. Available: School closures caused by Coronavirus (Covid-19) (unesco.org).

32. Aristovnik, A., Keržič, D., Ravšelj, D., et al. (2020). Impacts of the COVID-19 pandemic on life of higher education students: A global perspective. Sustain., 12(20), 1-34. https://doi.org/10. 3390/su12208438.

33. "COVID-19 vaccinations: Top countries." https://covidvax.org/. Accessed 17 April 2021.

34. Shang, J., Wan, Y., Luo, C., et al. (2020). Cell entry mechanisms of SARS-CoV-2. Proceedings of the National Academy of Sciences USA, 117(21), 1-8. https://doi.org/10.1073/pnas.2003138117.

Publisher's Note Springer Nature remains neutral with regard to jurisdictional claims in published maps and institutional affiliations. 